Tourism Research Journal

E-ISSN: 2598-9839

2019, Vol. 3 No. 2

\title{
Computational Gastronomy Use of Computing Methods in Culinary Creativity
}

\author{
Durga Prasad Shukla1, Pawan Ailawadi ${ }^{2}$ \\ SGT University ${ }^{1}$, Lovely Professional University ${ }^{2}$ \\ durga.sharad.shukla@gmail.com \\ pawanailawadi@yahoo.com
}

\begin{abstract}
Nowadays in culinary practices there is a great variety of regional cuisine in line with the cultural diversity of a place. This raises the question of whether there is a common pattern in determining the combination of food ingredients or the same principles in making recipes and producing certain tastes. For example, Western cuisine tends to use ingredients that have many flavor compounds, while East Asian cuisine tends to avoid ingredients sharing compounds. This study is driven by the results of previous studies showing the science of gastronomy is developing and producing the domain of computational gastronomy which contributes to the creation of recipes in innovative and creative ways. The purpose of this study is to understand the use of computational gastranomy in diverse food network in culinary creativity. The research design is exploring and looking for relationships between various aspects of food such as taste, aroma, seasoning, food ingredient, texture, and cooking methods. Cross sectional and longitudinal research are also carried out to find a causal relationship between computational gastronomy and culinary creation. The results showed that there was a relationship between various aspects, namely computational gastronomy, culinary creations, food content, and the use of technology in creating food recipes. The application of computational creativity in the culinary field can increase business value in the hotel kitchen area, the creation of recipes and food science.
\end{abstract}

Keywords: Computing, Cuisine, Gastronomy, Menu, Recipe. 


\section{A. Introduction}

"Can we compute gastronomy?", "How can we Network food?", "What would be the feasible impact of computing gastronomy in area of culinary creativity?"

Computational creativeness is an emerging part of artificial intelligence that uses computers in the creative process. A computational system that creates flavorful, innovative, and healthy culinary recipes by drawing on different data techniques (Canals et.al, 2016). The research will be an effort to justify the computational gastronomy through usage of more empirical data by way of generating modal based techniques. It is only going to build up pace with coverage of the large base and variables in era to come.

1. The recent and upcoming cultural diversity of culinary practice, determine the various ingredient combinations used or principles that transcend in the individual tastes and recipes.

2. The domain to introduce a flavor network that captures the flavor compounds shared by culinary ingredients, including the textures, aromas of the created recipe.

3. Given the increasing availability of information on food preparation, our data-driven investigation will open new avenues towards a systematic understanding of culinary practice (Ahn et al, 2011). The process used in haute cuisine establishments is both aligned and contradicts the model proposed in the culinary innovation literature, the general new product development model.

4. This study is motivated by the recent application of computational creativity in the culinary domain (Jagmohan et al, 2014). Particular the growing commercial importance of data-driven computation, to discover and offer unified framework in domains to which computational creativity can be applied and yield business value. The three fields are hotel kitchens, recipe creations and science

The rapidly growing body of publicly available data on food science and food usage may be utilized to analyze the network analysis along with the data mining in relation with gastronomy. Various kind of data driven components like flavors network, ingredients network based on food pairing through the use of computation leading to culinary creativity. Cooking without losing its art has become scientific and subject to various formulae mostly empirical in nature to a method of precision and not leaving anything to chance concept (Tallab and Alrazgan, 2016).

\section{Developing ingredient flavor network - Conceptual framework}

Flavor network According to Ahn et al, 2011:

A. The ingredients which are contained in two recipes (left column), together with the flavor compounds known to be present in the ingredients (right column). 
B. We obtain the flavor network by the ingredient-compound bipartite network into the ingredient space, whose nodes are ingredients, linked if they share at least one flavor compound. The thickness of links in the diagram represent the number of flavor compounds the two ingredients share and the size of each circle corresponds to the prevalence of the ingredients in recipes.

C. The delivery of recipe size, capturing the amount of ingredients per recipe, through the five cuisines

D. The frequency-rank plot of ingredients across the five cuisines show an approximately invariant distribution across cuisines.

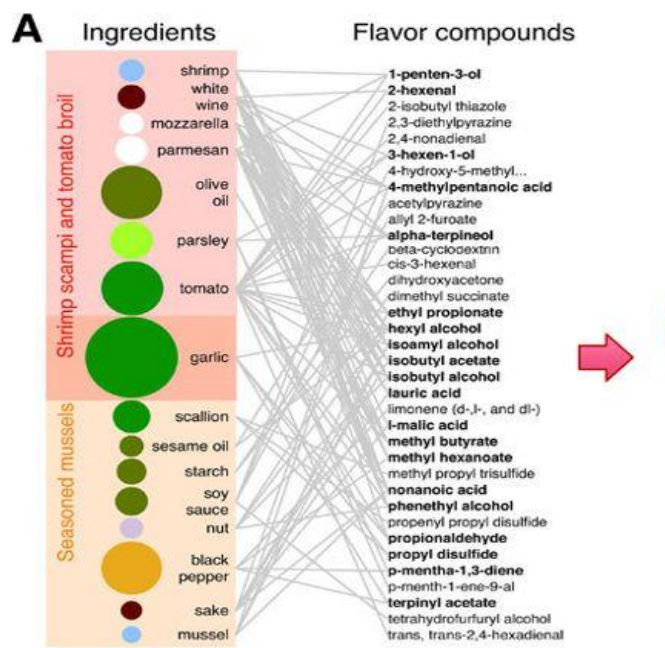

$$
\text { B Flavor network }
$$
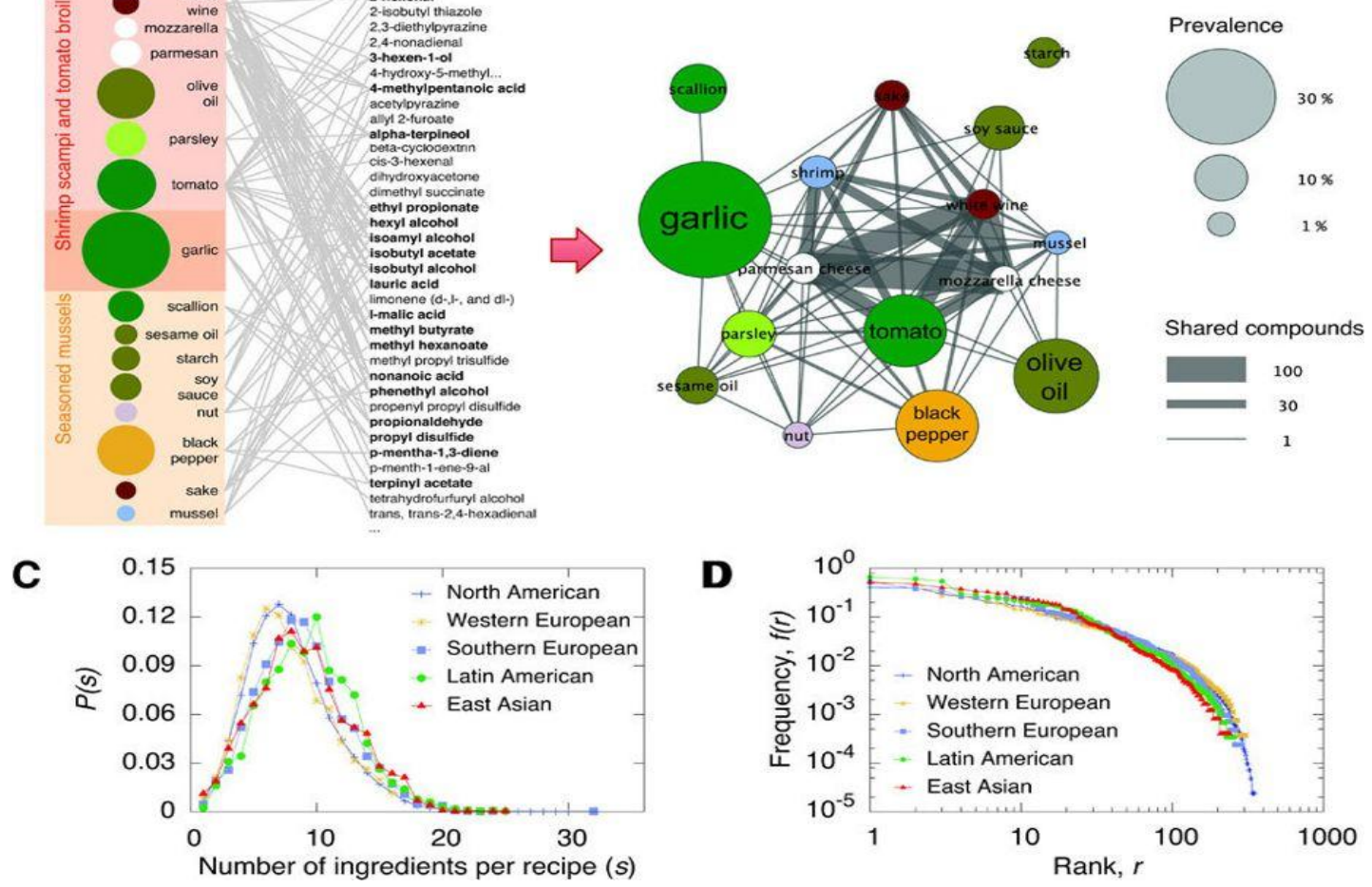

Source: Ahn et al, 2011

\section{B. Literature Review}

According to Spence et.al (2017) the human sense of taste and smell have become a target for interaction design and Human-Computer Interaction (HCI) research. Even though various connected fields such as sensory science, chemistry, biology, and molecular gastronomy have innovative our understanding on sensation and perception, the cooperative involvements that can be created based on taste and smell is relatively unexplored within HCI (Obrist et.al, 2017). "What do a Data Analyst \& a Chef have in common?". It is observed that very recently the study has started to understand the dimensions and features of taste and smell for multisensory experience design (Obrist et.al, 2016).

Gastronomy as a term is the art or science of specialized food involving 
experties. The ever growing enriched data on various food ingredients, their chemical composition and pairing combinations, in addition to the application of data mining, and knowledge extraction techniques, caused the emergence of a new field called Computational Gastronomy (Ahnert, 2013).

The data analytical study done highlights the statistical properties of the Indian regional cuisines, it brings out their specific culinary fingerprints that are used to design algorithms in order to facilitate and generate novel recipes along with recipe recommender systems. It actually forms a basis for further exploring possible causal relation between diet, health and a prospection of therapeutic uses of food ingredients. The study also provides deep exploring insights as to how large data base can change the way we perceive and look at food (Jain, Rakhi, Bagler, 2015).

While data of most cuisines followed uni-modal distribution, significantly as per the findings Mughlai cuisine showed a strong bimodal distribution which relatively had recipes with large sizes when compared with other cuisines.. This could be a defining indicator of the fact that Mughlai is derivative of an exotic royal flair. To understand and acknowledge the ingredient usage pattern, ingredients were ranked according to decreasing usage frequency within each cuisine. It was observed that all cuisines showed strikingly similar usage of ingredient profile which reflected the pattern of Indian cuisine. While indicating a growth in generic culinary mechanism, the distributions also indicated that certain ingredients are used excessively in various cuisines depicting their inherent relativity to 'fitness' or popularity within the regional cuisine sample (Jain, Rakhi, Bagler, 2015).

It give emphasis to ethnic and the cultural feature of a cuisine play a prominent role in deciding the ingredients of a recipe moderately than the actual chemistry of the ingredients. A research was directed on various flavor pairings in medieval European cuisine and the outcome revealed that the food pairing was found to be very positive in European recipes (Tallab and Alrazgan, 2016).

The study conducted on testing the hypothesis was published which focused on the Indian cuisine. The authors worked to quantify the present food pairing pattern and constructed models so as to find features that clarify the statistical properties of the particular cuisine. The bottom line was that the more the number of shared compounds between any two ingredients of Indian recipe, the lesser their co-occurrence probability (Tallab and Alrazgan, 2016).

The penultimate stage is selecting the best idea(s). Previous attempts at computational creativity have oft been criticized for not influencing selection, e.g. the painter AARON generates 150 pieces a night, but $\mathrm{H}$. Cohen selects which 5 to print by observing them all. (Pinel, 2014)

Strategy and idea culinary creations using the data analysis is strongly proposed as the first step in the development process and both parts perform interchangeably or simultaneously. Strategy will guide the development of an innovative dish. Michelin-starred chefs regarded local food product as being the 
basis for strategy or idea (reported by 12/12 interviewees )( Ottenbacher a \& Robert J. Harrington, 2008, downloaded by Temple University Libraries On: 21 November 2014).

In our system, data analytics allow the computer to predict which generated recipes will be the most surprising to a human observer, will be perceived as the most flavorful, and will have the best pairings of ingredients. These metrics are then used to rank the computationally generated ideas after which human makes the final selection. In our experiences, humans usually select one of the top three or four ideas rather than considering hundreds or thousands of possibilities. Hence selection is factually a collaboration between human and machine (Varshney, 2014). The final stage of creativity is externalizing. In a recipe creation system, this involves coming up with not just the list of ingredients (the focus of idea generation including selection), but also various proportions and recipe steps. Professional chefs with their elaborate experience often operate without computer support for externalizing, but amateurs appreciate guidance since it too requires significant creativity (Pinel , 2014).

A recent attempt in culinary computational creativity (Varshneyetal, 2013; Pinel and Varshney 2014) motivates the work presented in this paper. The system described in that study can create novel and flavorful recipes as perceived by people. It gathers data from culinary science establishes evaluation metrics based on codified expert knowledge in recipe design and human flavor perception, and can create quintillions of recipes which are far beyond the number of existing recipes. In fact, (Morris et al. 2012) discussed an approach of recipe creation system which was restricted to soups, stews and chili. The evolved culinary computational creativity system (Varshney et al. 2013; Pinel and Varshney 2014) is more general at present and has a cognitive flavor assessment component which is motivated by the scientific study of human flavor perception. The recipes created by this system have been served in multiple venues and have been well received. An independent assessment was done by Wired (Davis 2013) of a recipe which was created by the system concluded that "while the IBM dessert tasted better, it was also insanely elaborate, so we'll call it a draw." A hypothesis, which over the past few decades which have received attention among some various chefs and food scientists around the globe, states that ingredients with similar flavor compounds are more likely to blend well together than ingredients that do not. The findings revealed a dynamic synthesis of similarities and differences used by various European and US chefs (Ottenbacher and Harrington, 2009). Similarities included sequential and top down process and included a stress on the importance of a closely knitted connected network within culinary innovation. Key differences which were highlighted included the level of involvement throughout the process, the level at which technology was extensively used in the process, the understanding level of customer focused decisions, and also the likelihood of utilizing test kitchens or food scientists in 
the research and development for innovation process.

\section{Research Methods \\ Research-Gap}

With the past studies it was observed that food science along with gastronomy are likely to be the next beneficiaries and adopt the large-scale data analysis, thereby resulting in fields such as 'computational gastronomy' in contributing towards the recipe creation in innovative and creative manner, however has been an underexplored area. This study will bring out the various computational gastronomy creations which can be explored further and a modal may be evolved keeping the nutrition and dietary requirements which are a need of the day.

\section{Objective}

To understand the use of Computational Gastronomy along with various food networks in culinary creativity.

\section{Relevance of study}

The computing technology should include the fundamental social issues such as healthcare, leisure, labor, education, and food to build an ideal society. The main objective of this research is to bring together on a common platform of basic understanding amongst different stakeholders such as local food producers, chefs, home cooks, designers, engineers, data scientists, and sensory scientists so as to discuss and implicate the multifaceted nature of computing technology along with detailed culinary aspects with an advanced understanding of the intricacies involved in computational gastronomy.

\section{Research question}

How can culinary creativity be a part of computation covering various aspects of gastronomy?

What are the methods involved in computational gastronomy and culinary creativity?

\section{Hypothesis}

According to the "Hypothesis on The Food Pairing," certain ingredients compliment well with each other if they similar chemical composition is present. Now to do the judging whether the food pairing hypothesis is true or not, many researchers were immensely interested to validate it across given the vast diversity of varied cuisines from around the world. "A null hypothesis exists in between the relationship of computational Gastronomy and culinary creations"

\section{Research Design}

The research design will be a mixed relationship between exploratory and causal relationship between the various food aspects like flavors, aromas, seasonings, ingredients, textures, method of cooking which will be data based and finding the computational manner to execute and evolve with culinary creations through the various permutation and combination of the networks. All this will also have an empirical computation done so as to know the degree of utilization in executing 
new innovative recipes. A thorough cross sectional and longitudinal research will be conducted and the findings monitored on the basis of the causal relationship between computational gastronomic and culinary creations.

\section{Analysis and findings}

Large-scale computational data analysis provides a way to examine and connect them to each other, like for example, to understand and analyze the relationship between chemical data and recipes (Ahn et al., 2011). It is these network and data connections between different types of food variables that can provide us with a introspection on the study of our culinary culture by computing it.

Through the study of culinary culture through recipes online and social media. First, online recipe data is usually digital, which makes it easy to obtain and process large amounts. Next, online recipes often have rich metadata about the recipes. Along with the list of ingredients and instructions, recipes very often contain information such as type of cuisine, tags (e.g., seasons or holidays), cooking time involved, or macronutrient composition ( fats, carbohydrates and protein content). Such data can be used to study questions about food, culture, and health (Mouritsen, 2017).

With the advent and mass usage of information technology, there has been a total transformation in the way we prepare, perceive, think, manner of consumption or even talk about food. There are many varied types of foodrelated data available that are being collected on an enormous scale on various categories ranging from basic nature of food to a variety of chemical and nutritional data and extending up to recipe collections and social media postings. Large-scale computational data analysis provides a way to examine and connect them to each other, like for example, to understand and analyze the relationship between chemical data and recipes. It is these network and data connections between different types of food variables that can provide us with a introspection on the study of our culinary culture by computing it.

The research can be tested through more quantitative analysis with use of the food technology experiments for better understanding and Info tech in coming up with the devise for relating Human Computer Interface, however is going to take a considerable period of time. The research can also be explored in areas of easy, fast and simple breakfast preparation which is nutritional for women of India to lead healthy living through well devised and well computed recipes for the Women in India

\section{E. Conclusion}

The research study done elaborates on the various aspects concerning the relation between computational gastronomy, culinary creations, varied food ingredients components, advent and use of technology in recipe formulations along with the in-depth core competencies which need to ne networked amongst 
the stakeholders at all levels of food industry such as from the local producers to the ultimate processors like chefs, scientists and others. With all the data available from different sources the computation of the gastronomy analysis numerous food ingredient, flavor combinations that can be used in day to day working of preparation of menus keeping the nutrition count integrated along with the various preparation or processing units both industrial and hospitality sector in innovations through culinary creations.

\section{REFERENCES}

Ahn, Yong-Yeol \& Ahnert, Sebastian \& Bagrow, James \& Barabasi, AlbertLaszlo. (2011). Flavor network and the principles of food pairing. Scientific Reports. 1. 10.1038/srep00196.

Anupam Jain, Rakhi N K, Ganesh Bagler, Analysis of Food Pairing in Regional Cuisines of India, October 2, 2015

Ahnert, Sebastian E. 2013. Network analysis and data mining in food science: the emergence of computational gastronomy Flavour. vol. 2, no. 1, p. 4.

Canals, Alicia Bosch , Pere Grima Cintas, Diego Díaz López Business Analysis of a Culinary Innovation Project, January 2016

C. Spence, M. Obrist, C. Velasco, and N. Ranasinghe. 2017. Digitizing the chemical senses: Possibilities \&amp; pitfalls. International Journal of Human Computer Studies. https://doi.org/10.1016/j.ijhcs.2017.06.003

Davis, A. P. 2013. Digital gastronomy: When an IBM algorithm cooks, things get complicated-and tasty. Wired 20(10).

Jagmohan, A., Li, Y., Shao, N., Sheopuri, A., Wang, D., Varshney, L., Huang, P. 2014. Exploring application domains for computational creativity. In: Proceedings of the Fifth International Conference on Computational Creativity.

Jain A, Rakhi N K , Bagler G. (2015). Analysis of Food Pairing in Regional Cuisines of India. PLoS ONE 10(10): e0139539. https://doi.org/10.1371/journal.pone.0139539

Michael Ottenbacher \&Robert J. HarringtonThe Culinary Innovation Process- $A$ Study of Michelin-Starred Chefs, Journal of Culinary Science \& Technology Pages 9-35 | Published online: 22 Sep 2008,

Morris, R. G.; Burton, S. H.; Bodily, P. M.; and Ventura, D. 2012. Soup over beans of pure joy: Culinary ruminations of an artificial chef. Proc. Int. Conf. Comput. Creati. 119-125.

Mouritsen OG, Edwards-Stuart R, Ahn Y-Y and Ahnert SE (2017) Data-driven Methods for the Study of Food Perception, Preparation, Consumption, and Culture. Front. ICT 4:15. doi: 10.3389/fict.2017.00015

Network analysis and data mining in food science: the emergence of computational gastronomy 2013, 2:4 
Obrist, M., C. Velasco, C. Vi, N. Ranasinghe, A. Israr, A. Cheok, C. Spence, and P. Gopalakrishnakone. 2016. Sensing the future of HCI: Touch, taste, and smell user interfaces. Interactions 23, 5. https://doi.org/10.1145/2973568

Obrist, M., N. Ranasinghe, and C. Spence. 2017. Special issue: Multisensory human-computer interaction. International Journal of Human Computer Studies. https://doi.org/10.1016/j.ijhcs.2017.06.002

Obrist, Marianna, Marti, Patrizia, Velasco, Carlos, Tu, Yunwen, Narumi, Takuji and Holten Møller, Naja L The Future of Computing and Food, International Conference on Advanced Visual Interfaces, Castiglione della Pescaia, Grosseto, Italy, May 29 - June 01, 2018

Ottenbacher, Michael C. , Robert J. Harrington, Institutional, Cultural and Contextual Factors: Potential Drivers of the Culinary Innovation Process, July 1, 2009 Research Article

Pinel, F., and Varshney, L. R. 2014. Computational creativity for culinary recipes. Proc. ACM CHI Conf. on Human Factors in Computing Systems. To appear.

R.J.Harrington \&M.C.Ottenbacher, Managing the Culinary Innovation Process: The Case of New.Product,Development, Pages 4-18 | Received 30 Jul 2012, Accepted 27 Oct 2012, 19 Feb 2013

Shahad TalalTallab Muna Saleh Alrazgan, Exploring the Food Pairing Hypothesis in Arab Cuisine: A Study in Computational Gastronomy, Procedia Computer Science, Volume 82, 2016, Pages 135-137,

Tallab, S. T., and Alrazgan, M. S. (2016). Exploring the food pairing hypothesis in Arab cuisine: A study in computational gastronomy. Proc. Comput. Sci. 82, 135-137. doi: 10.1016/j.procs.2016.04.020

Varshney, L. R.; Pinel, F.; Varshney, K. R.; Bhattacharjya, D.; Schoergendorfer, A.; and Chee, Y.-M. 2013. A big data approach to computational creativity. arXiv:1311.1213.

Yong-Yeol Ahn, , Sebastian E. Ahnert, James P. Bagrow, \& Albert-László Barabási Flavor.network and the principles of food pairing, Scientific Reports volume1, 196 (2011) 